\title{
Effects of glutamate on dehydroascorbate uptake and Its enhanced vulnerability to the peroxidation in cerebral cortical slices
}

\author{
Jin Hyang Song ${ }^{1}$, Seon Ho Shin ${ }^{1}$ and III Min \\ Chung \\ 'Department of Physiology \\ Queen's University, Kingston \\ Ontario, Canada, K7L 3N6 \\ ${ }^{2}$ College of Life and Environment Science \\ Konkuk University, Seoul 143-701, Korea \\ ${ }^{3}$ Corresponding author: Tel, 82-2-450-3730; \\ Fax, 82-2-456-7856; E-mail, imcim@kkucc.konkuk.ac.kr
}

Accepted 23 November 2002

Abbreviations: AA, ascorbate; DHA, dehydroascorbate; GLUT, glucose transporter; GSH, glutathione; MDA, malondialdehyde; SVCT, sodium-dependent ascorbate transporter; TBARS, thiobarbituric acid-reactive substances

\begin{abstract}
Pro-oxidant properties of ascorbate have been studied with uses of brain tissues and neuronal cells. Here we address potential mechanism of ascorbate coupling with glutamate to generate oxidative stress, and the role which oxidized ascorbate (dehydroascorbate) transport plays in oxidative neuronal injury. Ascorbate in neurones can be depleted by adding glutamate in culture medium since endogenous ascorbate can be exchanged with glutamate, which enhances ascorbate/dehydroascorbate transport by depleting ascorbate in the neurons with the glutamate-heteroexchange. However, ascorbate is known readily being oxidized to dehydroascorbate in the medium. Glutamate enhanced the dehydroascorbate uptake by cells via a glucose transporter (GLUT) from extracellular region, and cytosolic dehydroascorbate enhanced lipid peroxide production and reduced glutathione (GSH) concentrations. Iso-ascorbate, the epimer of ascorbate was ineffective in generating the oxidative stress. These observations support the current concept that the high rates of dehydroascorbate transport via a GLUT after the release of ascorbate by glutamate leads to peroxidation, the role of glutamate on ascorbate/dehydroascorbate recycling being critical to induce neuronal death via an oxidative stress in
\end{abstract}

the brain injury.

Keywords: ascorbate; glucose transporter; glutathione; lipid peroxide; neuronal death; oxidative stress

\section{Introduction}

Ascorbate is an essential vitamin in human since primate cannot synthesize it due to lack of L-gulonolactone oxidase which is required for ascorbate biosynthesis, but it is needed to maintain the physiological function by ingestion from diets. The ascorbate is concentrated in particular organs such as adrenal gland (20 mM) and brain (1-2 mM) (Hornig, 1975). The brain contains about 10 times higher concentrations than circulating plasma concentration 10.1 mM) (Chatterjee et al., 1975; Spector, 1977). High concentrations in the brain suggest that ascorbate is actively transported through the uptake mechanisms from the circulating plasma ascorbate. However, the physiological role of high concentrations of ascorbate in the brain is not well known.

Ascorbate is generally considered to be a reducing agent since it is easily oxidized to dehydroascorbate, but it can also act as an oxidizing agent (Park et al., 1997; Song et al., 1999, 2001; Oh et al., 2002). The oxidative action involves rather complex reactions. Several mechanisms have been proposed to explain the oxidative action, but no particular mechanism is universally accepted. Three different mechanisms are known to be responsible for ascorbate transportation into the brain cells: sodium-dependent ascorbate transporter (SVCT), glucose transporter (GLUT) (May et al., 1998) and glutamate heteroexchange (Miele et al., 1994). The first SVCT route transports ascorbate with no accompanying redox action. However, the second GLUT route takes up dehydroascorbate, which is then reduced back to ascorbate this reduction process in turn generates oxidative stress in the neurons (Song et al., 1999, 2001).

Here we explored the possibility that glutamate heteroexchange (Miele et al., 1994), one of the major routes of ascorbate/dehydroascorbate transport, might be involved in the generation of oxidative stress during the ascorbate recycling. Ascorbate in neurons can be depleted by exposure to glutamate since mechanisms allow endogenous ascorbate to be exchanged with the glutamate. Depletion of endogen- 
ous ascorbate in neurons results in an increase in the uptake of ascorbate as dehydroascorbate since ascorbate in extracellular region is readily oxidized to dehydroascorbate under an oxygen supply. The resulting dehydroascorbate is taken up by a carrier (GLUT, a glucose transporter) mechanism into cells (Vera et al., 1993, 1995; Welch et al., 1995; Agus et al., 1997). Once inside the neuron dehydroascorbate is reduced back to ascorbate. This reduction process may be the cause of oxidative stress since oxidation-reduction requires an electron transfer from one compound to another. Therefore, rapid uptake of an oxidizing agent such as dehydroascorbate could induce damages of vital cellular components. Our observation will contribute to clarify the physiologic roles of ascorbate/dehydroascorbate in the brain cells.

\section{Materials and Methods}

\section{Chemicals}

Fetal bovine serum and RPMI 1640 medium was purchased from Bio-Rad Laboratories (Mississagua, Canada). Halothane was obtained from Halocarbon Laboratories (River Edge, NJ). Other chemicals were purchased from Sigma (St. Louis, MO). Plastic tissue culture dishes and tubes were purchased from Fisher Scientifics (Nepean, Canada).

\section{Cortical Slice Preparation}

Adult male SpragueDawley rats (300-325 g, Charles River Canada, Montreal) were used in these experiments. Rats were anesthetized lightly with halothane and were decapitated, and brain was removed from the skull rapidly and placed in ice-cold phosphate buffered saline (PBS, $\mathrm{pH} 7.4)$. The slices $(<1 \mathrm{~mm})$ were prepared from the cerebral cortex region of the brain, and then placed in an incubation tube containing a RPMI medium supplemented with $10 \%$ fetal bovine serum. Nine volumes of warm medium were then added and then kept between 36.5 and $36.7^{\circ} \mathrm{C}$ for $10 \mathrm{~min}$ before treatment with pharmacological agents. After $2 \mathrm{~h}$ incubation with pharmacological agents in shaking water bath, samples were centrifuged at $1,500 \mathrm{~g}$ for $2 \mathrm{~min}$ and the pellet was washed with PBS.

\section{Measurement of lipid peroxidation}

The lipid peroxidation product in brain homogenates was assayed by measurement of the malondialdehyde (MDA) concentration using the thiobarbituric acid reactive substances (TBARS) method. Brain homogenates $(0.5 \mathrm{mg}$ of protein $/ \mathrm{ml})$ were mixed with $2 \mathrm{ml}$ of $0.375 \%$ thiobarbituric acid, $15 \%$ trichloroacetic acid
(Sigma) and $0.04 \%$ butylatedhydroxytoluene in $0.25 \mathrm{M}$ $\mathrm{HCl}$, and the concentration of MDA was measured (Buege and Aust, 1978). The MDA concentration is expressed as nanomoles per milligram of protein using tetraethoxypropane as a reference standard. After a 30-min incubation, the reaction was centrifuged at $1,500 \mathrm{~g}$ for $10 \mathrm{~min}$ and the absorbance of supernatant was measured at $532 \mathrm{~nm}$.

\section{Determination of glutathione (GSH)}

In order to further support oxidative stress induced by ascorbate or dehydroascorbate, we have examined changes of glutathione concentration after treatment with $50 \mu \mathrm{M}$ glutamate combined with different concentrations $(0,100$ or $500 \mu \mathrm{M})$ of ascorbate, dehydroascorbate or isoascorbate. Brain GSH concentration was measured using a method described by Hissin and Hilf (1976). Cortical slices (250 mg) were homogenized with $0.5 \mathrm{ml}$ of $25 \%$ metaphosphoric acid $\left(\mathrm{Na}_{2} \mathrm{HPO}_{3}\right)$ and $2 \mathrm{ml}$ of $0.1 \mathrm{M}$ sodium phosphate$5 \mathrm{mM}$ EDTA buffer ( $\mathrm{pH}$ 8.0). After centrifugation at $15,000 \mathrm{~g}$ for $30 \mathrm{~min}$, the supernatants were incubated with o-phthalaldehyde for GSH determination. The GSH content was determined by a fluorometry with excitation and emission wave lengths of $350 \mathrm{~nm}$ and $420 \mathrm{~nm}$, respectively. Data were calculated on the basis of GSH calibration curves. In next series of experiments, concentrations of ascorbate, dehydroascorbate and isoascorbate were fixed at $300 \mu \mathrm{M}$, and different concentrations $(0,100$ and $1,000 \mu \mathrm{M})$ of glutamate were added in the culture medium containing the cortical slices.

\section{$\left[{ }^{14} \mathrm{C}\right]$ Dehydroascorbate uptake assay}

In order to examine the involvement of a GLUT transportation, the effects of the GLUT inhibitors, such as cytochalasin B and glucose on dehydroascorbate transport activity by measuring the uptake of radiolabeled compounds after treated with $\left[{ }^{14} \mathrm{C}\right]$ ascorbate $(10 \mu \mathrm{M} ; 7 \mathrm{mCi} / \mathrm{mmol})$ at $37^{\circ} \mathrm{C}$ was studied. Slices of cerebral cortex (containing $10 \mathrm{mg}$ protein) were incubated with $1 \mathrm{ml}$ of $10 \mu \mathrm{M}$ ascorbate containing $0.1 \mu \mathrm{Ci}\left[{ }^{14} \mathrm{C}\right.$ ]ascorbate $(8.60 \mathrm{mCi}$ per mmol, NEC 146 , Dupont NEN) in the presence or absence of $50 \mu \mathrm{M}$ glutamate for up to $5 \mathrm{~h}$ in a shaking water bath at $37^{\circ} \mathrm{C}$. To investigate the effect of GLUT inhibitor on $\left[{ }^{14} \mathrm{C}\right]$ dehydroascorbate (DHA) uptake, we choose the $10 \mu \mathrm{M}$ wortmannin (Sigma Chemicals) and $15 \mathrm{mM}$ D-glucose (Fisher Scientific), and amounts of $\left[{ }^{14} \mathrm{C}\right]$ uptake were determined after incubation for $5 \mathrm{~h}$. Slices were collected and washed twice with $2 \mathrm{ml}$ PBS ( $\mathrm{pH} \mathrm{7.4)} \mathrm{after} \mathrm{each} \mathrm{incubation} \mathrm{periods.} \mathrm{The}$ samples were homogenized and transferred to Scintiverse (Fisher Scientific). The radioactivities were 
counted with liquid scintillation counter (Beckman).

\section{Other procedures}

Protein concentrations were determined using the Lowry assay. Bovine serum albumin was used as a reference standard. The overall effects of the treatments are expressed as percent changes with respect to control conditions.

Results are presented as means \pm SEM. The GraphPad Prism (GraphPad, San Diego, CA) was used for all statistical analyses. Statistical significance of differences between values was evaluated by performing ANOVA followed by Bonferroni's multiple comparison test as a post-hoc analysis. Values of $P$ $<0.05$ were considered as significant.

\section{Results}

The effect of glutamate on oxidative stress induced by ascorbate was investigated (Figure 1). Neither treatments with up to $500 \mu \mathrm{M}$ ascorbate alone nor $50 \mu \mathrm{M}$ glutamate alone increased thiobarbituric acidreactive substances (TBARS) production in cerebral cortical slices indicating that they are subthreshold concentrations. However, TBARS concentrations were significantly elevated to $183 \pm 18 \%(P<0.01)$ and 166 $\pm 11 \%(P<0.05)$ by $300 \mu \mathrm{M}$ and $500 \mu \mathrm{M}$ ascorbate in the presence of $50 \mu \mathrm{M}$ glutamate, respectively (upper panel, Figure 1). These obsevations indicate that glutamate potentiates ascorbate-induced oxidative stress. Treatments with $500 \mu \mathrm{M}$ dehydroascorbate without glutamate elevated TBARS concentration to $135 \pm 10 \% \quad(P<0.05)$ (middle panel, Figure 1) in contrast to $500 \mu \mathrm{M}$ ascorbate $(98 \pm 22 \%)$ (upper panel, Figure 1). These observations illustrate that the dehydroascorbate has more potent stimulatory action on TBARS production than ascorbate. When we added $50 \mu \mathrm{M}$ glutamate in the incubation medium, both 300 and $500 \mu \mathrm{M}$ dehydroascorbate significantly elevated TBARS concentration to $165 \pm 18 \% \quad(P<$ $0.001)$ and $167 \pm 4 \%(P<0.001)$, respectively in the cortical slices (middle panel, Figure 1) showing similar pattern of the stimulatory action as glutamate's potentiation effect on ascorbate (upper panel, Figure 1).

In order to determine specificity of the optical isomer on the glutaminate's potentiation effect on isoascorbate-induced oxidative stress was also studied by measuring TBARS concentrations. The isoascorbate did not increase TBARS concentration with or without $50 \mu \mathrm{M}$ glutamate (lower panel, Figure 1). Isoascorbate is the epimer of ascorbate and its redox potential is the same as ascorbate, but isoascorbate did not show any stimulatory action on TBARS production. These negative results indicate that tertiary
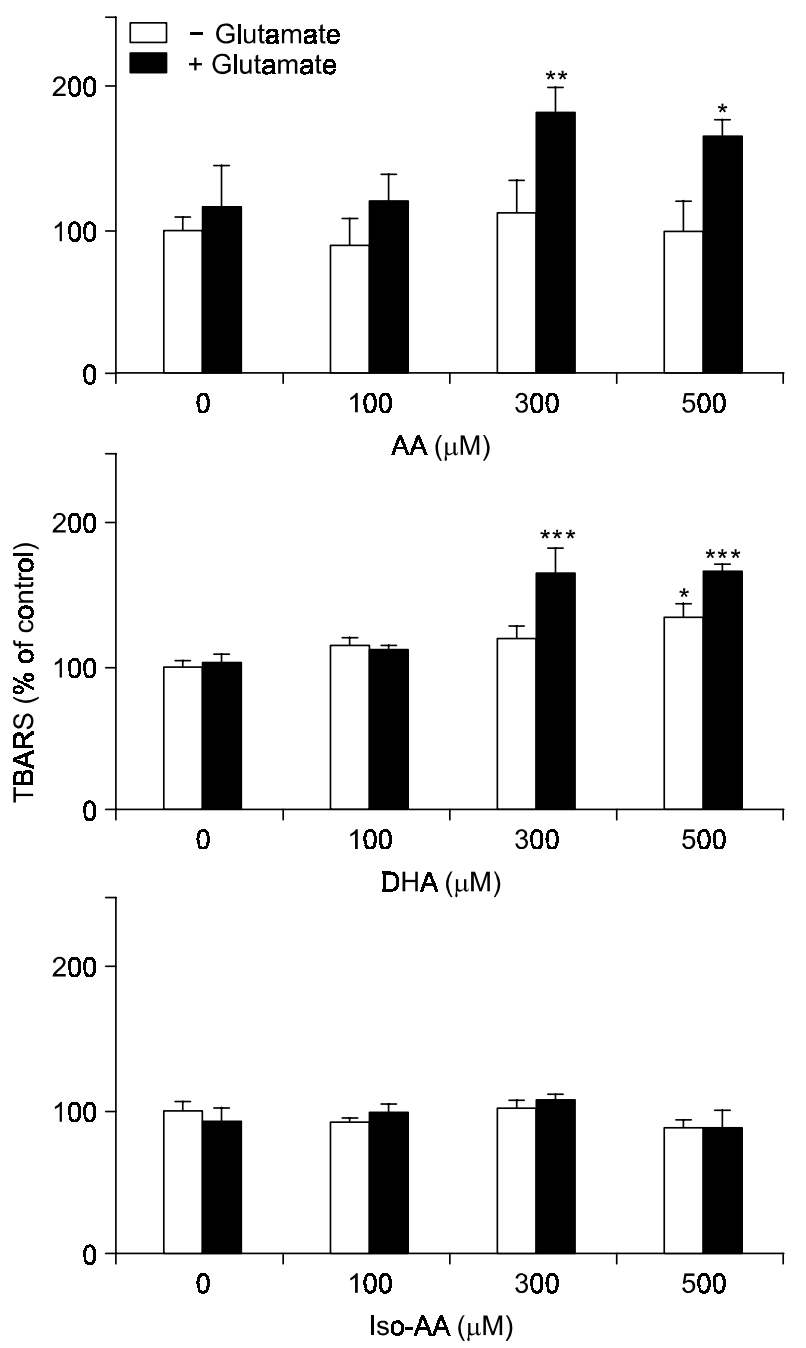

Figure 1. Effects of glutamate on the production of thiobarbituric acid reactive substances (TBARS) induced by ascorbate (AA), dehydroascorbate (DHA) or iso-ascorbate (Iso-AA). Cortical slices were pretreated without or with $50 \mu \mathrm{M}$ glutamate and subsequently incubated with one of either $A A, D H A$ or Iso-AA in a concentration dependent fashions for $2 \mathrm{~h}$. Quantities of TBARS (lipid peroxide) were expressed as equivalent of malondialdehyde values $(n=6)$. Each vertical bar represents the mean \pm SEM $(n=6) . *, P<0.05 ; * *, P<0.01 ; * * *$, $P<0.001$.

configuration of the molecular structure plays a determinant role on ascorbate-induced oxidative stress.

The concentration dependency of ascorbate, dehydroascorbate and isoascorbate was carried out with set level of $300 \mu \mathrm{M}$ under varying levels of glutamate $(0,100$ and $1,000 \mu \mathrm{M})$. The results showed that TBARS concentrations were not changed in the groups treated with ascorbate, dehydroascorbate or isoascorbate without glutamate, but groups treated with ascorbate or dehydroascorbate plus $100 \mu \mathrm{M}$ glutamate significantly elevated TBARS concentrations to 
$202 \pm 13 \%(P<0.001)$ or $224 \pm 15 \%(P<0.001)$, respectively (Figure 2). Ascorbate or dehydroascorbate treatment also elevated TBARS concentrations in the medium containing $1,000 \mu \mathrm{M}$ glutamate to $140 \pm 7 \%$ $(P<0.01)$ or $137 \pm 5 \%(P<0.01)$, respectively (Figure $2)$. The isoascorbate treatments did not change TBARS concentration with or without glutamate (Figure 2).

Possible affect of glutamate along with varying levels of ascorbate, dehydroascorbate or isoascorbate

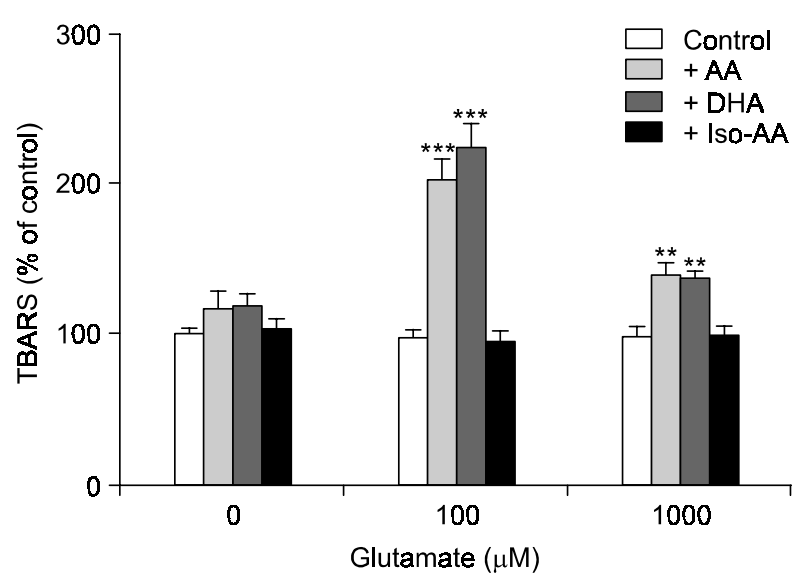

Figure 2. Concentration dependent effects of glutamate on the production of thiobarbituric acid reactive substances (TBARS) induced by ascorbate (AA), dehydroascorbate (DHA) or iso-ascorbate (Iso-AA). Cortical slices were pretreated with 0,100 or $1000 \mu \mathrm{M}$ glutamate and subsequently incubated with one of either AA, DHA or Iso-AA at 500 $\mu \mathrm{M}$ for $2 \mathrm{~h}$. Quantities of TBARS (lipid peroxide) were expressed as equivalent of malondialdehyde values $(n=6)$. Each vertical bar represents the mean \pm SEM $(n=6)$. **, $P<0.01 ; * * *, P<0.001$.

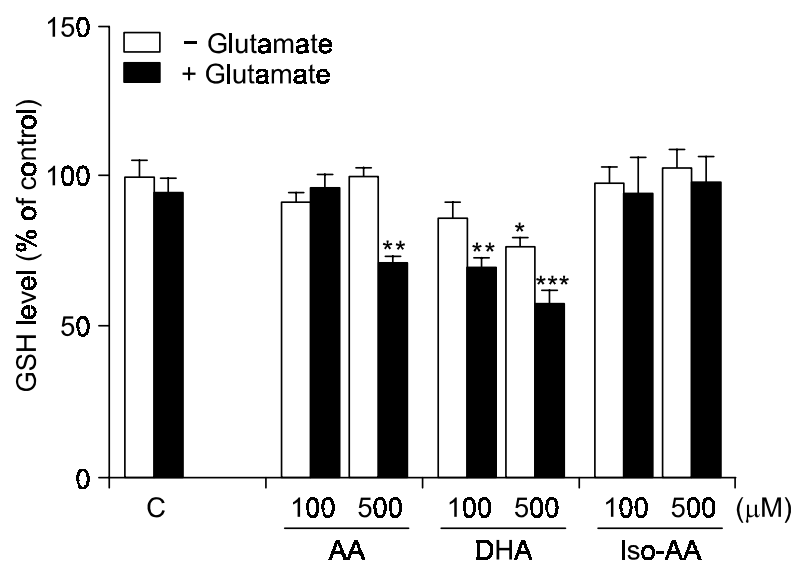

Figure 3. Effects of glutamate on the levels of endogenous glutathione (GSH) in the treatment of ascorbate (AA), dehydroascorbate (DHA) or iso-ascorbate (Iso-AA). Cortical slices were pretreated without or with $50 \mu \mathrm{M}$ glutamate and subsequently incubated with one of either $A A, D H A$ or Iso-AA in a concentration dependent fashions for $2 \mathrm{~h}$. Each vertical bar represents the mean $\pm \operatorname{SEM}(n=6) .{ }^{*}, P<0.05$; $* *, P<0.01 ; * * *, P<0.001$ on the glutathione concentration, another cellular redox participants was examined. Fifty micromole glutamate plus $100 \mu \mathrm{M}$ ascorbate was ineffective but higher concentration $(500 \mu \mathrm{M})$ of ascorbate decreased glutathione level in the cortical slices to $71 \pm 3 \%$ ( $P$ $<0.01$ ) (Figure 3). Both $50 \mu \mathrm{M}$ glutamate plus 100 or $500 \mu \mathrm{M}$ dehydroascorbate lowered glutathione concentration to $70 \pm 3 \%(P<0.01)$ or $58 \pm 4 \%(P<$ 0.001 ), respectively (Figure 3 ). These observations confirm that dehydroascorbate is more potent on reducing glutathione contents than ascorbate. The combined treatments with glutamate and isoascorbate were ineffective in decreasing glutathione concentration (Figure 3). The inverse relationships between TBARS and glutathione concentrations indicate that both glutatmate plus ascorbate or dehydroascorbate generates oxidative stress.

Study on the affect of the GLUT inhibitors, such
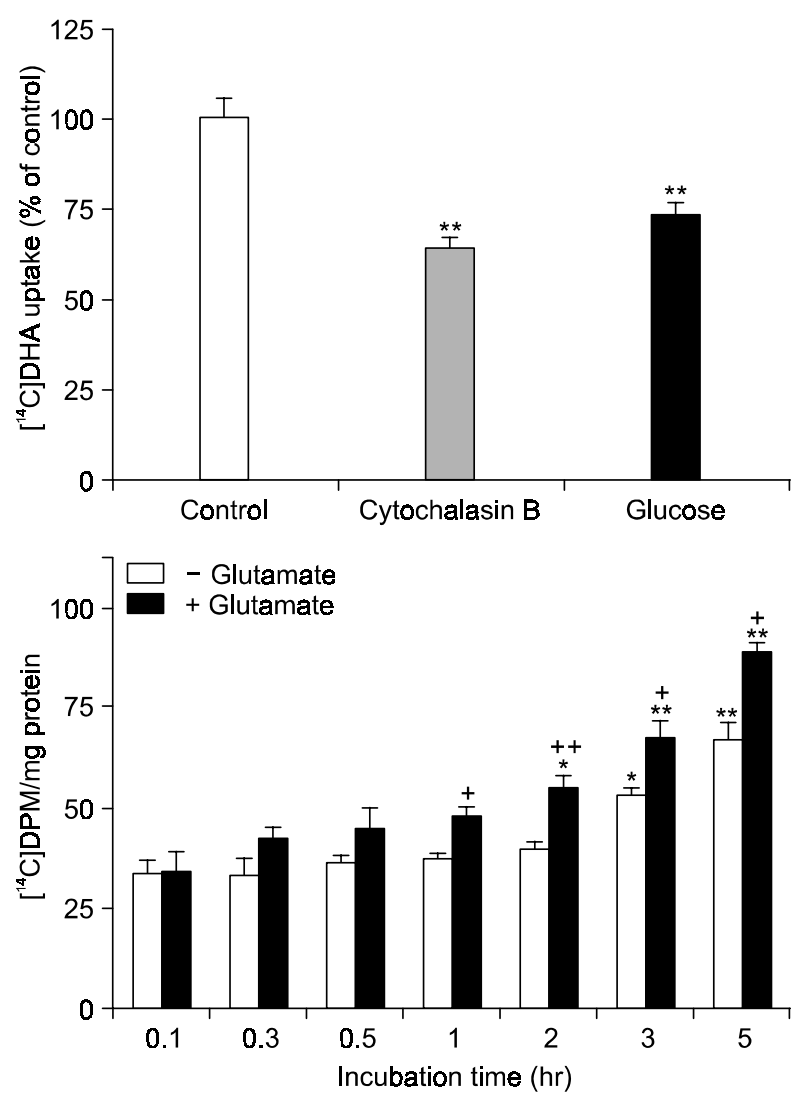

Figure 4. Effects of GLUT antagonists, such as cytochalasin B and glucose and glutamate on dehydroascorbate (DHA) transportation. To test DHA transporting activity via a GLUT, cortical slices were treated with $\left[{ }^{14} \mathrm{C}\right.$ lascorbate for $4 \mathrm{~h}$ in the absence or presence of $10 \mu \mathrm{M}$ cytochalasin B or $15 \mathrm{mM}$ glucose. Effects of glutamate $(50 \mu \mathrm{M})$ were evaluated after cortical slices treated with $\left[{ }^{T /} \mathrm{C}\right]$ ascorbate for up to $5 \mathrm{~h}$ periods. Each vertical bar represents means $\pm \operatorname{SEM}(n=6)$. *, $P<0.05 ; * *, P<0.01$ vs. control;,$+ P<0.05 ;++, P<0.01$ vs. non-glutamte treated group. 
as cytochalasin B and glucose on dehydroascorbate transport activity by measuring the uptake of radiolabeled compounds, showed that treatment of cortical slices with $10 \mu \mathrm{M}$ cytochalasin B or $15 \mathrm{mM}$ glucose resulted in significantly lower uptake of radiolabeled compound $(76 \pm 8 \%, P<0.05$ or $62 \pm 5 \%, P<0.01$ ) in the hepatocytes after $5 \mathrm{~h}$ incubation than the control $(100 \pm 5 \%)$. These observations support the concept that $\left[{ }^{14} \mathrm{C}\right]$ ascorbate is taken up through a GLUT after oxidation to $\left[{ }^{14} \mathrm{C}\right]$ dehydroascorbate as cytochalasin B or glucose suppressed uptake of the labeled compound (Figure 4A). To investigate whether glutamate enhances the dehydroascorbate transport to cells from extracellular region, kinetics of dehydroascorbate transport activity was examined in the absence or presence of glutamate. In the absence of glutamate, the level of dehydroascorbate uptake remained the same for at least $2 \mathrm{~h}$ but gradually increased next $3 \mathrm{~h}$ period (Figure 4B). Treatment of cortical slices with $50 \mu \mathrm{M}$ glutamate increased the rate of dehydroascorbate uptake during 1 to $5 \mathrm{~h}$ incubation periods (Figure 4B). Taken together, these observations indicate that glutamate stimulates dehydroascorbate transport via a GLUT.

\section{Discussion}

Ascorbic acid is a well established reducing agent, but also known to increase the oxidation potential and induce oxidative injury in cells (Leung et al., 1993; Sakagami and Satoh, 1997; Si et al., 1998; Song et al., 1999). We have been trying to establish the mechanism for ascorbate-induced oxidative stress in tissues (Si et al., 1998; Song et al., 1999, 2001). The present study is designed to elucidate a part of ascorbate recycling which generates the oxidative stress. One could hypothesize some reasonable path for ascorbate-induced oxidative stress based on the numerous observations reported. Tissues containing high concentrations of ascorbate such as the brain (1-2 $\mathrm{mM}$ ) release ascorbate during hypoxia (Hornig, 1975; Matsuo et al., 1995). Energy supply is required to maintain a high concentration of ascorbate in a tissue. The released ascorbate is oxidized to dehydroascorbate in plasma (or medium) since sufficient amounts of molecular oxygen $\left(\mathrm{O}_{2}\right)$ are available in plasma ( $\mathrm{Pa}_{02}$ 95 \pm 2 torr, Walker et al., 1997) to oxidize the ascorbate, and the dehydroascorbate is present in plasma (Dhariwal et al, 1991; Will and Bayers, 1996). The dehydroascorbate is taken up by neurons via a transporter (GLUT) (Agus et al., 1997). The higher concentration of dehydroascorbate in neurons may be resulted from the facts that dehydroascorbate competes with higher blood glucose concentrations on the lesser numbers of GLUT which transports both glucose and dehydroascorbate into cells. These observations of higher dehydroascorbate concentrations are consistent with a concept that dehydroascorbate and glucose compete for the same carrier mechanism, 'glucose (or dehydroascorbate) transporter' (GLUT).

The GLUT is known to be a common carrier for glucose and dehydroascorbate and is an efficient transporter (Angulo et al., 1998). It has been reported that GLUT-dependent uptake mechanism of dehydroascorbate, an oxidized form of ascorbate (Vera et al., 1993) in various cell lines as well as tissues. The dehydroascorbate taken up by cells is efficiently reduced to ascorbate by enzymatic processes (Bode et al., 1993; May et al., 1998) and by chemical reduction-oxidation reaction. The reduction of a compound means oxidation of other cellular components such as glutathione and unsaturated lipids. When the glutathione in cells is decreased or depleted, electrons will be taken from vital components which trigger a cascade reaction of apoptosis.

It has been known that the cerebral cortex contains several millimolar concentrations of ascorbate and there is greater than 10 -fold difference between the concentration of ascorbate in neurons and glia, postulating the presence of a dynamic regulation mechanism to transport ascorbate into the cortical neurons. Several investigators have observed that the extracellular ascorbate concentration is modulated dynamically by glutamate transport, where an increase in ascorbate concentration is caused by heteroexchange with glutamate (Miele et al., 1994) as glutamate is taken up by transporter(s). In the present study, we tried to demonstrate that glutamate-heteroexchange as a route of ascorbate uptake are involved in ascorbate/dehydroascorbate recycling of neuronal cells.

The working hypothesis in this study is that glutamate causes the accelerated release of endogenous ascorbate into the medium, resulting in a 'physiological' concentration (equivalent to the ascorbate concentration in the brain) of ascorbate in the medium bring oxidized to dehydroascorbate by molecular oxygen $\left(\mathrm{O}_{2}\right)$, which is then efficiently transported back into the neurons. Furthermore, the glutamate-induced depletion of ascorbate enhances rates of dehydroascorbate uptake via the GLUT to maintain homeostasis. The GLUT transporter functions independently of the glutamate heteroexchange route. The transport of dehydroascorbate into the neurons generates oxidative stress. This oxidative stress is commonly visualized with increasing quantities of TBARS and decreasing concentrations of glutathione. The observations that glutamate's potentiation to ascorbate- or dehydroascorbate-induced oxidative stress, support our proposed mechanism that high uptake rates of dehydroascorbate generate oxidative stress. 
In addition, our hypothesis is supported by the observation that ascorbate consistently elevated lipid peroxide production in liver slices whereas isoascorbate did not (Figure 1). Both ascorbate and isoascorbate (the epimer of ascorbate) have the same chemical structure and the same red-ox potential. The main difference is the tertiary structure at the first asymmetric carbon (optical isomer). Isoascorbate has vitamin $\mathrm{C}$ activities, but its potency is much weaker (approximately $10 \%$ of ascorbate). It was also observed that isoascorbate is ineffective on TBARS production with or without glutamate (Figure 1). The ineffectiveness of isoascorbate supports that (i) red-ox potential of ascorbate does not generate oxidative stress since ascorbate and isoascorbate have the same red-ox potential, and (ii) 'dehydroascorbate' transporter (GLUT) is stereo-specific which can differentiate optical isomers. These negative results of isoascorbate are consistent with our proposed mechanism that ascorbate-induced oxidative stress is generated by high uptake rates of dehydroascorbate via GLUT.

In this study, $100 \mu \mathrm{M}$ and $500 \mu \mathrm{M}$ of ascorbate or dehydroascorbate are at subthreshold concentrations on TBARS production and on reduction of glutathione concentrations (Figure 3). It was seen that $50 \mu \mathrm{M}$ glutamate was unable to potentiate $100 \mu \mathrm{M}$ ascorbate, but potentiated $500 \mu \mathrm{M}$ ascorbate on reduction of glutathione concentration (Figure 3). However, $50 \mu \mathrm{M}$ glutamate potentiated effects of both 100 and $500 \mu \mathrm{M}$ dehydroascorbate on the reduction of glutathione concentration (Figure 3). The fact that dehydroascorbate is more potent than ascorbate is also consistent with our hypothesis since ascorbate should be oxidized to dehydroascorbate before it can enhance TBARS production and cause reduction of glutathione concentrations. In addition, total quantities of dehydroascorbate and its uptake rates are two important factors we must consider. Large quantities of ascorbate can be taken up either directly by ascorbate or indirectly via dehydroascorbate. Obviously, direct uptake of ascorbate cannot generate oxidative stress since ascorbate cannot take an extra-electron. Dehydroascorbate can be taken up in large quantities without generating oxidative stress if the uptake rate is slower than synthesis rate of glutathione. The newly synthesized glutathione would easily absorb the oxidative potential generated by dehydroascorbate without depleting glutathione pool when its uptake rate is slow. Only high uptake rates of dehydroascorbate in sufficient quantities generate oxidative stress and initiate apoptosis.

During a stroke (or hypoxia), ascorbate is released from neurons. The neurons attempt to restore ascorbate to physiological concentrations when they receive the normal concentrations of nutrients includ- ing oxygen during a reperfusion period. Massive influx of dehydroascorbate via a GLUT can occur during the reperfusion period after a stroke.

Collectively, the observations suggest that the massive influx of dehydroascorbate (oxidized ascorbate) through a GLUT coupling with glutamate-heteroexchange will generate oxidative stress causing brain damage.

\section{References}

Agus DB, Gambhir SS, Pardridge WM, Spielholz C, Baselga J, Vera JC, Golde DW. Vitamin C crosses the blood-brain barrier in the oxidized form through the glucose transporters. J Clin Invest 1997;100:2842-8

Angulo C, Rauch MC, Droppelmann A, Reyes AM, Slebe JC, Delgado-Lopez F, Guaiquil VH, Vera JC, Concha II. Hexose transporter expression and function in mammalian spermatozoa: cellular localization and transport of hexoses and vitamin C. J Cell Biochem 1998;71:189-203

Bode AM, Yavarow CR, Fry DA, Vargas T. Enzymatic basis for altered ascorbic acid and dehydroascorbic acid levels in diabetes. Biochem Biophys Res Commun 1993;91:1347-53

Buege JA, Aust SD. Microsomal lipid peroxidation. Methods Enzymol 1978;52:302-10

Chatterjee IB, Majumder AK, Nandi BK, Subramanian N. Synthesis and some major functions of vitamin $C$ in animals. Ann NY Acad Sci 1975;258:24-47

Dhariwal KR, Hartzell WO, Levine M. Ascorbic acid and dehydroascorbic acid measurements in human plasma and serum. Am J Clin Nutr 1991;54:712-6

Hissin PJ, Hilf R. A fluorometric method for determination of oxidized and reduced glutathione in tissues. Anal Biochem $1976 ; 74: 214-26$

Hornig D. Distribution of ascorbic acid, metabolites and analogues in man and animals. Ann NY Acad Sci 1975; 258:103-18

Leung PY, Mayashita K, Young M, Taso CS. Cytotoxic effect of ascorbate and its derivatives on cultured malignant and nonmalignant cell lines. Anticancer Res 1993;13:475-80

Matsuo $\mathrm{Y}$, Kihara $\mathrm{T}$, Ikeda M, Ninomiya M, Onodera $\mathrm{H}$, Kogure K. Role of neutrophils in radical production during ischemia and reperfusion of the rat brain: effect of neutrophil depletion on extracellular ascorbyl radical formation. J Cereb Blood Flow Metab 1995;15:941-7

May JM, Cobb CE, Mendiratta S, Hill KE, Burk RF. Reduction of the ascorbyl free radical to ascorbate by thioredoxin reductase. J Biol Chem 1998;273:23039-45

Miele M, Boutelle MG, Fillenz M. The physiologically induced release of ascorbate in rat brain is dependent on impulse traffic, calcium influx and glutamate uptake. Neuroscience 1994;62:87-91

Oh SI, Lee MS, Kim Cl, Song KY, Park SC. Aspartate modulates the ethanol-induced oxidative stress and glutathione 
utilizing in rat testes. Exp Mol Med 2002;34:47-52

Park YC, Oh SI, Park YH, Park SC. Modulation by aspartate of ischemia/reperfusion-induced oxidative stress in rat liver. Exp Mol Med 1997;29:19-23

Sakagami H, Satoh K. Prooxidant action of two antioxidants: ascorbic acid and gallic acid. Anticancer Res 1997;17:221-4

Si F, Ross GM, Shin SH. Glutathione protects PC12 cells from ascorbate- and dopamine-induced apoptosis. Exp Brain Res 1998;23:263-8

Song JH, Shin SH, Ross GM. Prooxidant effects of ascorbate in rat brain slices. J Neurosci Res 1999;58:328-36

Song $\mathrm{JH}$, Shin SH, Ross GM. Oxidative stress induced by ascorbate causes neuronal damage in an in vitro system. Brain Res 2001;895:66-72

Spector R. Vitamin homeostasis in the central nervous system. N Engl J Med 1977;296:1393-8
Vera JC, Rivas $\mathrm{Cl}$, Fischbarg J, Dolde GW. Mammalian facilitative hexose transporters mediate the transport of dehydroascorbic acid. Nature 1993;364:79-82

Vera JC, Rivas $\mathrm{Cl}$, Velasquez FV, Zhang RH, Concha II, Golde DW. Resolution of the facilitated transport of dehydroascorbic acid from its intracellular accumulation as ascorbic acid. J Biol Chem 1995;270:23706-12

Walker JK, Lawson BL, Jennings DB. Breath timing, volume and drive to breathe in conscious rats: comparative aspects. Respir Physiol 1997;107:241-50

Welch RW, Wang Y, Crossman A, Park JB, Kirk KL, Levine $M$. Accumulation of vitamin $C$ (ascorbate) and its oxidized metabolite dehydroascorbic acid occurs by separate mechanisms. J Biol Chem 1995;270:12584-92

Will JC, Bayers T. Does diabetes mellitus increase the requirement for vitamin C? Nutr Rev 1996;54:193-202 